\title{
Coupled out of plane vibrations of spiral beams for micro-scale applications
}

\author{
M. Amin Karami ${ }^{\mathrm{a}, *}$, Bulent Yardimoglu ${ }^{\mathrm{b}}$, Daniel J. Inman ${ }^{\mathrm{c}}$ \\ a Department of Engineering Science and Mechanics, Virginia Tech, Blacksburg, VA 24061, USA \\ ${ }^{\mathrm{b}}$ Department of Mechanical Engineering, Izmir Institute of Technology, Izmir, Turkey \\ ${ }^{\mathrm{c}}$ Center for Intelligent Material Systems and Structures, Department of Mechanical Engineering, Virginia Tech, Blacksburg, VA 24061, USA
}

\section{A R T I C L E I N F O}

\section{Article history:}

Received 20 October 2009

Received in revised form

12 July 2010

Accepted 14 July 2010

Handling editor: S. Ilanko

Available online 12 August 2010

\begin{abstract}
A B S T R A C T
An analytical method is proposed to calculate the natural frequencies and the corresponding mode shape functions of an Archimedean spiral beam. The deflection of the beam is due to both bending and torsion, which makes the problem coupled in nature. The governing partial differential equations and the boundary conditions are derived using Hamilton's principle. Two factors make the vibrations of spirals different from oscillations of constant radius arcs. The first is the presence of terms with derivatives of the radius in the governing equations of spirals and the second is the fact that variations of radius of the beam causes the coefficients of the differential equations to be variable. It is demonstrated, using perturbation techniques that the derivative of the radius terms have negligible effect on structure's dynamics. The spiral is then approximated with many merging constant-radius curved sections joined together to approximate the slow change of radius along the spiral. The equations of motion are formulated in non-dimensional form and the effect of all the key parameters on natural frequencies is presented. Non-dimensional curves are used to summarize the results for clarity. We also solve the governing equations using Rayleigh's approximate method. The fundamental frequency results of the exact and Rayleigh's method are in close agreement. This to some extent verifies the exact solutions. The results show that the vibration of spirals is mostly torsional which complicates using the spiral beam as a host for a sensor or energy harvesting device.
\end{abstract}

(c) 2010 Elsevier Ltd. All rights reserved.

\section{Introduction}

We are motivated to look at the vibrations of spiral shaped structures as a prelude to sensing and energy harvesting using the piezoelectric effect in micro-electro-mechanical systems (MEMS) devices. Vibrational energy harvesters convert vibrations available in the environment to electrical energy. The energy generated can be used to power sensor nodes [1]. These energy self-sufficient sensors can be placed in remote places to gather data and wirelessly transmit information. A key challenge in designing MEMS energy harvesters is to make them resonate with low frequency ambient vibrations. Spiral geometry has been suggested as a possible option for compact low frequency substrate in energy harvesting devices [2]. However the vibrational analysis of curved beam with varying radius (spirals) is missing in the literature [3]. Hu et al. [3] approximated the spiral by eccentric constant-radius arcs but only derived the governing equations and did not solve them for vibration characteristics. This paper attempts to solve the free vibrations of spiral beams and pave the way to the modeling of spiral MEMS harvesting devices.

\footnotetext{
${ }^{*}$ Corresponding author. Tel.: +1540 8086840 .

E-mail addresses: karami@vt.edu (M. Amin Karami), bulentyardimoglu@iyte.edu.tr (B. Yardimoglu), dinman@vt.edu (D.J. Inman).
} 


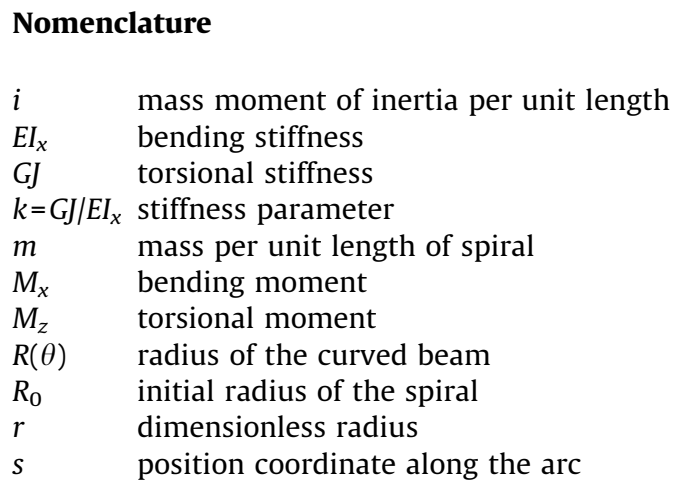

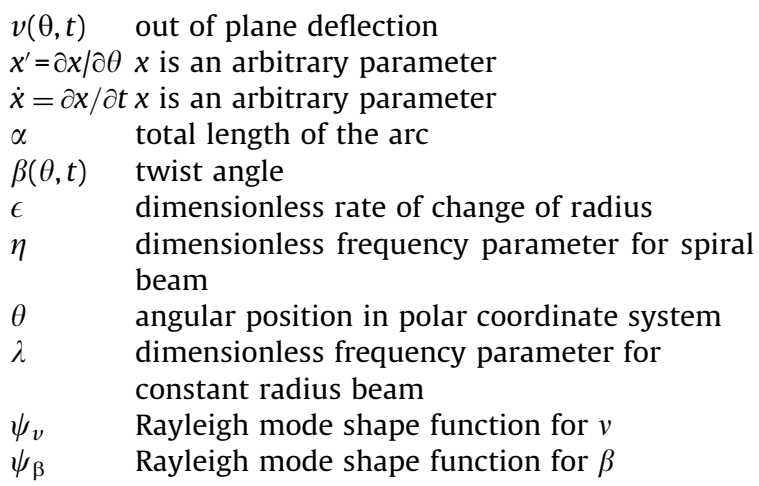

Out-of plane vibrations of circular curved beams have been studied by many investigators. Here we focus our attention on just out-of-plane vibrations of curved beams having variable radius of curvature. In historical order: Love [4] derived the equations of curved beams of arbitrary geometry. Chang and Volterra [5] obtained the upper and lower bounds of the first four natural frequencies of elastic clamped arcs of which the center lines were in the forms of circles, cycloids, catenaries, and parabolas by using a method based on differential operator theory. Wang [6] employed the Rayleigh-Ritz method to predict the natural frequency of clamped elliptic arc. Irie et al. [7] determined the steady state response of a Timoshenko curved beam with circular, elliptical, catenary and parabolical neutral axes driven at the free end by use of the transfer matrix approach. Huang et al. [8] presented the dynamic responses of non-circular Timoshenko curved beam with viscous damping by using a numerical Laplace transform approach. They considered the numerical examples for a two-span elliptic beam subjected to a rectangular impulse. Then, Huang et al. [9] developed a dynamic stiffness matrix by using the Laplace transform technique for both the free vibration and forced vibration of non-uniform parabolic curved beams with various ratios of rise to span. Tufekci and Dogruer [10] obtained the exact solution of the differential equations for the static behavior of an arch with varying curvature and cross section including the shear deformation effect by using the initial value method.

Despite the long history of attempts to solve for the vibrations of arcs, a straight forward solution of the vibration of spiral beams, suitable for a design procedure, is still unavailable. In this paper we study the dynamic behavior of spiral beams. The fact that the radius of the beam slowly changes along the spiral justifies the use of perturbation methods and discretization of the beam. The governing differential equations and corresponding boundary conditions are derived simultaneously from Hamilton's principle. A new general solution is proposed for the curved beam with constant radius. Next, the effect of terms including the rate of change of radius $\left(R^{\prime}\right)$ are shown to be negligible using the method of multiple scales. Subsequently, to tackle change of radius in spirals, the beam is approximated with several merged constant radius arcs and the vibrations of the resulting approximation is studied. We non-dimensionalize the governing equations and solve them to find the natural frequencies of the spiral beam. The dimensionless frequencies are functions of arc length, rate of change of radius and the stiffness ratio. The effects of each of these parameters on the resonant frequencies are studied. The natural frequency and mode shapes of a clamped-free full turn spiral as well as the mode shapes of a five turn spiral are next calculated and plotted. The alternate approach of Rayleigh's method is also used to verify the results of the exact solution. Finally, the important application issue of whether or not the out of plane vibrations of spirals are dominantly bending or torsional is considered.

\section{Governing equations}

The moment-displacement relationships for the spiral depicted in Fig. 1 are given by Love [4] as follows:

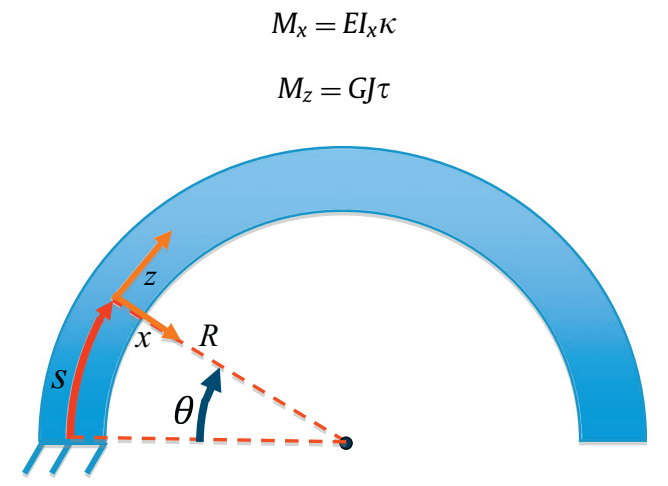

Fig. 1. Coordinates. 
where

$$
\begin{gathered}
\kappa=\frac{\beta}{R}-\frac{\partial^{2} v}{\partial s^{2}} \\
\tau=\frac{\partial \beta}{\partial s}+\frac{1}{R} \frac{\partial v}{\partial s}
\end{gathered}
$$

The strain energy $U$ and the kinetic energy $T$ of the curved beam for out-of-plane motion are

$$
\begin{gathered}
U=\frac{1}{2} \int_{0}^{S_{L}}\left(M_{x} \kappa+M_{z} \tau-Y v-\Phi \beta\right) d s \\
T=\frac{1}{2} \int_{0}^{S_{L}}\left(m \dot{v}^{2}+i \dot{\beta}^{2}\right) d s
\end{gathered}
$$

Here, $i$ is the mass moment of inertia per unit length of the curved beam. In Eq. (5), $Y$ and $\Phi$ are the external force and the twisting moment, respectively. For the free vibration problem, neglecting rotational inertia, Hamilton's principle, Eqs. (5) and (6) along with Eqs. ((1)-(4)) give the equations of motion as

$$
\begin{gathered}
\frac{\partial^{2}}{\partial s^{2}}\left[E I_{x}\left(\frac{\beta}{R}-\frac{\partial^{2} v}{\partial s^{2}}\right)\right]+\frac{\partial}{\partial s}\left[\frac{G J}{R}\left(\frac{\partial \beta}{\partial s}+\frac{1}{R} \frac{\partial v}{\partial s}\right)\right]=m \ddot{v} \\
\frac{E I_{x}}{R}\left(\frac{\partial^{2} v}{\partial s^{2}}-\frac{\beta}{R}\right)+\frac{\partial}{\partial s}\left[G J\left(\frac{\partial \beta}{\partial s}+\frac{1}{R} \frac{\partial v}{\partial s}\right)\right]=0
\end{gathered}
$$

The boundary conditions associated with equations of motion are:

$$
\begin{gathered}
\left.\frac{E I_{x}}{R}\left(\frac{\partial^{2} v}{\partial s^{2}}-\frac{\beta}{R}\right) \delta\left(\frac{\partial v}{\partial s}\right)\right|_{0} ^{S_{L}}=0 \\
\left.G J\left(\frac{\partial \beta}{\partial s}+\frac{v}{R}\right) \delta \beta\right|_{0} ^{S_{L}}=0 \\
\left.\left\{\frac{\partial}{\partial s}\left[E I_{x}\left(\frac{\beta}{R}-\frac{\partial^{2} v}{\partial s^{2}}\right)\right]+\frac{G J}{R}\left(\frac{\partial \beta}{\partial s}+\frac{1}{R} \frac{\partial v}{\partial s}\right)\right\} \delta v\right|_{0} ^{S_{L}}=0
\end{gathered}
$$

In the above equations, the arc length differential, $d s$, is

$$
d s=\sqrt{R^{2} d \theta^{2}+d R^{2}}=R d \theta \sqrt{1+\epsilon^{2}}
$$

where dimensionless rate of change of radius is defined as

$$
\epsilon=\frac{1}{R} \frac{d R}{d \theta}
$$

\section{Vibration of a constant radius curved beam}

Next consider finding the natural frequencies and mode shapes of a curved beam with constant radius. The solution is based on the one presented by Ojalvo [11] with a different choice of the form of the assumed solution. Assuming $R$ is constant in Eq. (7), one gets the following governing differential equations in terms of the twist angle and out of plane deflection:

$$
\begin{gathered}
v^{i v}-R \beta^{\prime \prime}-k\left(v^{\prime \prime}+R \beta^{\prime \prime}\right)=-\frac{\rho A R^{4}}{E I_{x}} \ddot{v} \\
v^{\prime \prime}-R \beta+k\left(R \beta^{\prime \prime}+v^{\prime \prime}\right)=0
\end{gathered}
$$

where the stiffness parameter $k$ is defined as $k=G J / E I$. Evaluating $v^{\prime \prime}$ from Eq. (13b) and substituting that in Eq. (13a) we can derive

$$
\beta^{(4)}+2 \beta^{\prime \prime}+\beta=\frac{\rho A R^{4}}{G J} \frac{1+k}{R} \ddot{v}
$$

The separation of variables is now used to solve the above partial differential equation by assuming

$$
\beta(\theta, t)=B(\theta) T(t), v(\theta, t)=V(\theta) T(t)
$$


Substituting for $\beta$ and $v$ from Eqs. (15) into (14) and grouping the terms which depend on the same variable together results,

$$
\frac{B^{(4)}+2 B^{\prime \prime}+B}{V}=\frac{\rho A R^{4}}{G J} \frac{1+k}{R} \frac{\ddot{T}}{T}=-\lambda \frac{1+k}{R}
$$

The expression $-\lambda 1+k / R$ is a constant not depending on either $\theta$ or $t$. The right side of Eq. (16) prescribes a harmonic variation for $T, T=C \cos \left(\omega_{n} t+\phi\right)$. The natural frequency depends on the value of the dimensionless parameter $\lambda$, given by

$$
\omega_{n}^{2}=-\frac{\ddot{T}}{T}=\frac{G J}{\rho A R^{4}} \lambda
$$

Eq. (16) can be rewritten as

$$
B^{(4)}+2 B^{\prime \prime}+B+\lambda \frac{1+k}{R} V=0
$$

We need to differentiate the above equation twice to be able to use Eq. (13b) to have the equation in terms of only $B$ :

$$
B^{(6)}+2 B^{(4)}+B^{\prime \prime}+\lambda \frac{1+k}{R} V^{\prime \prime}=0
$$

Evaluating $V^{\prime \prime}$ in terms of $B$ from Eq. (13b) gives

$$
B^{(6)}+2 B^{(4)}+(1-k \lambda) B^{\prime \prime}+\lambda B=0
$$

Here we deviate from [11] as we consider an exponential form of general solution:

$$
B=\sum_{i=1}^{6} A_{i} e^{s_{i} \theta}
$$

where $s_{i}$ is one of the complex roots of the characteristic equation, Eq. (22). Each $A_{i}$ is a constant complex number. Thus

$$
s^{6}+2 s^{4}+(1-k \lambda) s^{2}+\lambda=0
$$

Once $B$ is calculated the bending function $V$ can be correspondingly evaluated by twice integration of Eq. (13b):

$$
V=\sum_{i=1}^{6} \frac{R}{k+1}\left(\frac{1}{S_{i}^{2}}-k\right) A_{i} e^{s_{i} \theta}
$$

The essential boundary conditions at the clamped end and the natural boundary conditions at the free end can be written in terms of $B, V$ and their derivatives. The resulting six equations can be written in a matrix form:

$$
[M]_{6 \times 6}\left[\begin{array}{c}
A_{1} \\
\vdots \\
A_{6}
\end{array}\right]=0_{6 \times 1}
$$

Here the matrix $[M]$ depends on the values of the roots, $s_{i}$ which are in turn related to $\lambda$ by Eq. (22). Eq. (24) yields the trivial solution, $\left[A_{1} \ldots A_{6}\right]^{T}=0$, unless $\operatorname{det}([M])=0$. For specific eigenvalues, $\lambda=\lambda_{n}, \operatorname{det}([M])=0$ vanishes and the problem can have nontrivial solutions. The natural frequencies of the system are determined by these eigenvalues using Eq. (17) [12].

The main distinction of this method compared to [11] is that we do not assume that each of the general solutions are real. The $s_{i}$ are complex and the same is true for any $A_{i} e_{i}^{s}$. When all added up the solution will be real. This makes it unnecessary to categorize the solutions of Eq. (22) based on the sign of each root and consider them separately (as done in [11]).

The complex approach here was validated by calculating the natural frequencies of a clamped-clamped constant radius arc and comparing the values to previously published results. The results precisely matched with those in [11].

\section{The effect of terms containing $R^{\prime}$ on the vibration of spirals}

The radius of Archimedean spiral varies linearly with the polar angle, $\theta: R=R_{0}+a \theta$, as depicted in Fig. 2 . This implies that that the rate of change of radius, $R^{\prime}$, is constant and is equal to $a$. Since the radius changes along the beam, we redefine dimensionless rate of change or radius as $\epsilon=R^{\prime} / R_{0}$, where $R_{0}$ is the initial radius of the spiral. We also assume that the rate of change of radius is small compared to the radius, i.e. $\epsilon \ll 1$. Then we approximate Eq. (11) by writing its Taylor expansion about $\epsilon=0$, resulting in

$$
d s=R(\theta) d \theta\left(1+\left[\frac{2 \epsilon}{2 \sqrt{1+\epsilon^{2}}}\right]_{\epsilon=0} \epsilon+O\left(\epsilon^{2}\right)\right)
$$




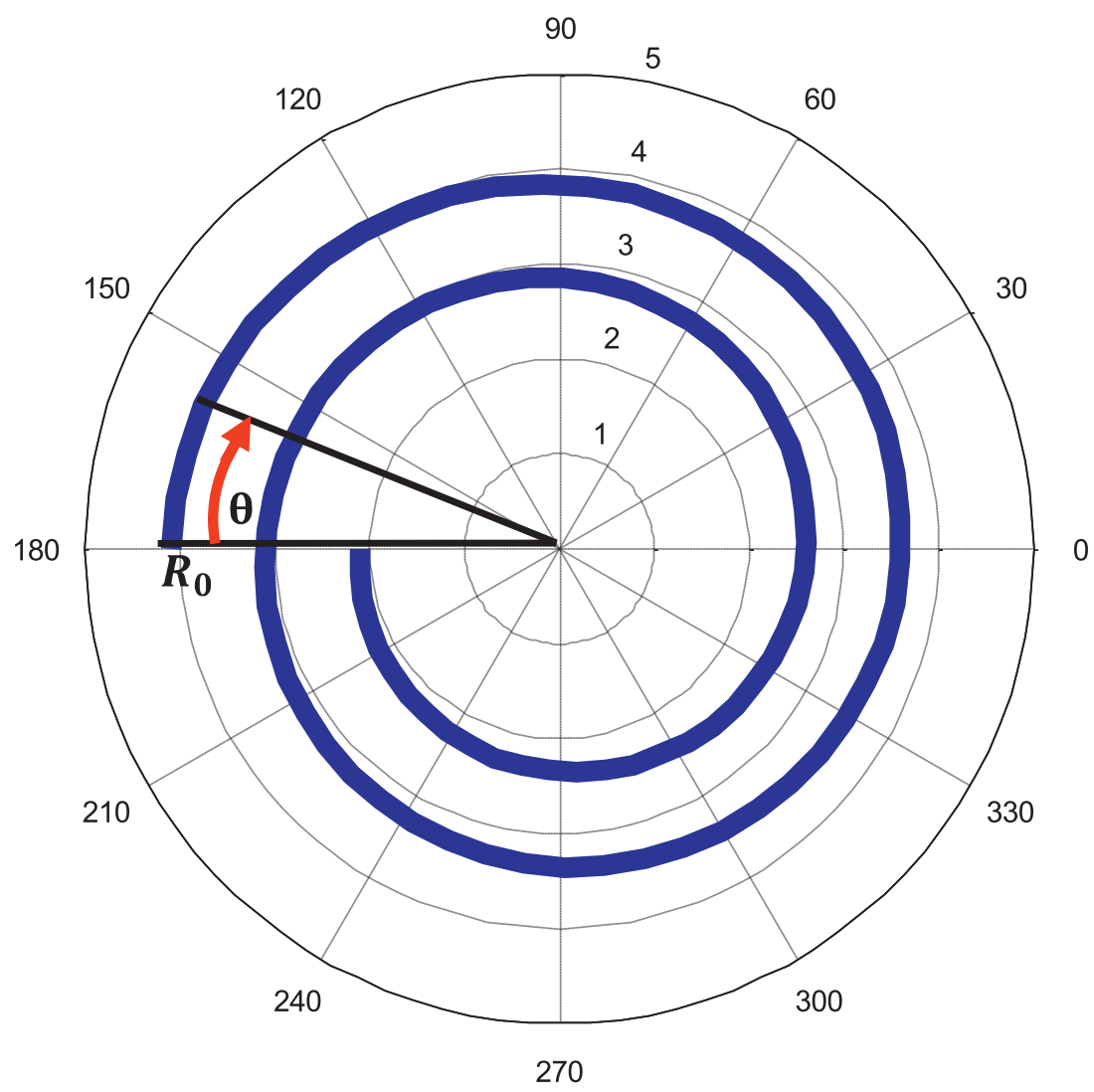

Fig. 2. Archimedean spiral.

Thus to the first approximation we have $d s=R d \theta$. To derive the governing equation for the Archimedean spiral we first exchange the derivatives with respect to $s$ with derivatives with respect to $\theta$ as

$$
\begin{gathered}
d s=R(\theta) d \theta, \quad \frac{\partial f}{\partial s}=\frac{1}{R(\theta)} \frac{\partial f}{\partial \theta}, \quad \frac{\partial^{2} f}{\partial s^{2}}=\frac{1}{R(\theta)^{2}} \frac{\partial^{2} f}{\partial \theta^{2}}-\frac{R^{\prime}}{R(\theta)^{3}} \frac{\partial f}{\partial \theta} \\
\frac{\partial^{3} f}{\partial s^{3}}=\frac{1}{R(\theta)^{3}} \frac{\partial^{3} f}{\partial \theta^{3}}-\frac{3 R^{\prime}}{R(\theta)^{4}} \frac{\partial^{2} f}{\partial \theta^{2}}, \quad \frac{\partial^{4} f}{\partial s^{4}}=\frac{1}{R(\theta)^{4}} \frac{\partial^{4} f}{\partial \theta^{4}}-\frac{6 R^{\prime}}{R(\theta)^{5}} \frac{\partial^{3} f}{\partial \theta^{3}}
\end{gathered}
$$

Evaluating all the derivatives in Eqs. (7a) and (7b) using Eq. (26) results in the two governing equations for Archimedean spiral beams. Using separation of variables, similar to previous section, results in

$$
\begin{gathered}
R V^{(4)}-6 R^{\prime} V^{(3)}-k R V^{\prime \prime}+3 k R^{\prime} V^{\prime}-(k+1) R^{2} B^{\prime \prime}+(2 k+3) R R^{\prime} B^{\prime}=\gamma R^{5} V \\
k R^{2} B^{\prime \prime}-k R R^{\prime} B^{\prime}-R^{2} B+(k+1) R V^{\prime \prime}+(1-2 k) R^{\prime} V^{\prime}=0 \\
\omega_{n}^{2}=\frac{E I}{\rho A} \gamma
\end{gathered}
$$

Eqs. (27) have two major differences with Eq. (13). The first is the existence of terms including $R^{\prime}$. The second, which is quite fundamental, is that $R$ in Eqs. (27) is no longer a constant but rather varies as a function of $\theta$. We tackle these complexities one by one. In this section we study how the terms with $R^{\prime}$ affect the natural frequencies while assuming $R$ is still a constant. Physically this study applies to very short spirals, in which the radius varies but since the spiral is very short the radius will be almost the same everywhere. Next Eqs. (27) are rewritten in terms of the dimensionless parameters $\lambda=R^{4} / k \gamma$ and $\epsilon=R^{\prime} / R_{0}$. This definition of $\lambda$ is consistent with Eq. (17). This results in

$$
\begin{gathered}
V^{(4)}-6 \epsilon V^{(3)}-k V^{\prime \prime}+3 k \epsilon V^{\prime}-(k+1) R B^{\prime \prime}+(2 k+3) R \epsilon B^{\prime}=k \lambda V \\
k R B^{\prime \prime}-k R \epsilon B^{\prime}-R B+(k+1) V^{\prime \prime}+(1-2 k) \epsilon V^{\prime}=0
\end{gathered}
$$

Since the rate of change of radius is small compared to the radius, perturbation methods can be used to analyze the vibrations of the spiral. We use the method of multiple scales [13] to solve Eq. (28). First we expand the solution of our 
system as

$$
V=v_{0}+\epsilon v_{1}+\ldots, \quad B=B_{0}+\epsilon B_{1}+\ldots
$$

$v_{0}$ and $B_{0}$ are the solution of Eq. (28) provided $\epsilon=0$. The terms $\epsilon V_{1}$ and $\epsilon B_{1}$ can be interpreted as the effect of small perturbations on the solution. In fact, the dependency of $V$ and $B$ on $\epsilon$ and $\theta$ occurs over different scales namely $\theta, \epsilon \theta, \epsilon^{2} \theta$ and so on. $\epsilon \theta$ is a slower scale than $\theta$ since it becomes notable only when $\theta$ is big. We determine $V$ and $B$ as functions of the scales, $T_{0}=\theta$ and $T_{1}=\epsilon \theta$, rather than functions of $\epsilon$ and $\theta$. We define:

$$
D_{0}=\frac{\partial}{\partial T_{0}}, D_{0}^{2}=\frac{\partial^{2}}{\partial T_{0}^{2}}, \quad \text { and } \quad D_{1}=\frac{\partial}{\partial T_{1}}, D_{1}^{2}=\frac{\partial^{2}}{\partial T_{1}^{2}}
$$

All the derivatives in Eqs. (28) with respect to $\theta$ must be reevaluated using the chain rule [13] in terms of derivatives of $T_{0}$ and $T_{1}$ resulting in

$$
\begin{aligned}
& 3 R \epsilon\left(D_{0} B_{0}\right)+2 k R \epsilon\left(D_{0} B_{0}\right)+3 k \epsilon\left(D_{0} v_{0}\right)-R\left(D_{0}^{2} B_{0}\right)-k R\left(D_{0}^{2} B_{0}\right)-R \epsilon\left(D_{0}^{2} B_{1}\right)-k R \epsilon\left(D_{0}^{2} B_{1}\right)-k\left(D_{0}^{2} v_{0}\right)-k \epsilon\left(D_{0}^{2} v_{1}\right) \\
& -6 \epsilon\left(D_{0}^{3} v_{0}\right)+D_{0}^{4} v_{0}+\epsilon\left(D_{0}^{4} v_{1}\right)-2 R \epsilon\left(D_{0} D_{1} B_{0}\right)-2 k R \epsilon\left(D_{0} D_{1} B_{0}\right)-2 k \epsilon\left(D_{0} D_{1} v_{0}\right)+4 \epsilon\left(D_{0}^{3} D_{1} v_{0}\right)-k \lambda v_{0}-k \epsilon \lambda v_{1}=0
\end{aligned}
$$

and

$$
\begin{gathered}
-k R \epsilon\left(D_{0} B_{0}\right)+\epsilon\left(D_{0} v_{0}\right)-2 k \epsilon\left(D_{0} v_{0}\right)+k R\left(D_{0}^{2} B_{0}\right)+k R \epsilon\left(D_{0}^{2} B_{1}\right)+D_{0}^{2} v_{0}+k\left(D_{0}^{2} v_{0}\right)+\epsilon\left(D_{0}^{2} v_{1}\right) \\
+k \epsilon\left(D_{0}^{2} v_{1}\right)+2 k R \epsilon\left(D_{0} D_{1} B_{0}\right)+2 \epsilon\left(D_{0} D_{1} v_{0}\right)+2 k \epsilon\left(D_{0} D_{1} v_{0}\right)-R B_{0}-R \epsilon B_{1}=0
\end{gathered}
$$

Since the above equations must hold for arbitrary values of $\epsilon$, in each of the equations the coefficients of the like powers of $\epsilon$ must satisfy the identities:

$$
D_{0}^{4} v_{0}-R\left(D_{0}^{2} B_{0}\right)-k\left[D_{0}^{2} v_{0}+R\left(D_{0}^{2} B_{0}\right)\right]-k \lambda v_{0}=0
$$

and

$$
\begin{gathered}
D_{0}^{4} v_{1}-R\left(D_{0}^{2} B_{1}\right)-k\left[D_{0}^{2} v_{1}+R\left(D_{0}^{2} B_{1}\right)\right]-k \lambda v_{1}=-3 R\left(D_{0} B_{0}\right)-2 k R\left(D_{0} B_{0}\right) \\
-3 k\left(D_{0} v_{0}\right)+6\left(D_{0}^{3} v_{0}\right)+2 R\left(D_{0} D_{1} B_{0}\right)+2 k R\left(D_{0} D_{1} B_{0}\right)+2 k\left(D_{0} D_{1} v_{0}\right)-4\left(D_{0}^{3} D_{1} v_{0}\right)
\end{gathered}
$$

also

$$
D_{0}^{2} v_{0}-R B_{0}+k\left[R\left(D_{0}^{2} B_{0}\right)+D_{0}^{2} v_{0}\right]=0
$$

and

$$
\begin{gathered}
D_{0}^{2} v_{1}-R B_{1}+k\left[R\left(D_{0}^{2} B_{1}\right)+D_{0}^{2} v_{1}\right]=k R\left(D_{0} B_{0}\right)-D_{0} v_{0}+2 k\left(D_{0} v_{0}\right) \\
-2 k R\left(D_{0} D_{1} B_{0}\right)-2\left(D_{0} D_{1} v_{0}\right)-2 k\left(D_{0} D_{1} v_{0}\right)
\end{gathered}
$$

We can solve Eqs. (31a1) and (31b1) for $v_{0}$ and $B_{0}$. The equations are the same as Eq. (13) and they can be written in the similar exponential form:

$$
B_{0}\left(T_{0}, T_{1}\right)=\sum_{i=1}^{6} A_{i}\left(T_{1}\right) e^{s_{i} T_{0}}, \quad v_{0}\left(T_{0}, T_{1}\right)=\sum_{i=1}^{6} \frac{R}{k+1}\left(\frac{1}{S_{i}^{2}}-k\right) A_{i}\left(T_{1}\right) e^{s_{i} T_{0}}
$$

The exponents, $s_{i}$, are the roots of the following algebraic equation:

$$
s^{6}+2 s^{4}+(1-k \lambda) s^{2}+\lambda=0
$$

Next, we substitute for $B_{0}$ and $v_{0}$ in the left sides of Eqs. (31a2) and (31b2). To be able to identify the secular terms the resulting two equations must be combined into one. For this purpose we differentiate Eq. (31a2) twice with respect to $\theta$ so that it is composed of $D_{0}^{2} v_{1}$ or higher derivatives of $v_{1}$ with respect to $T_{0}$. Eq. (31b2) is then used to write all $v_{1}$ related terms in terms of $B_{1}$ and its derivatives. The left side of the resulting equation is $B_{1}{ }^{(6)}+2 B_{1}{ }^{(4)}+(1-k \lambda) B_{1}{ }^{(2)}+\lambda B_{1}=\ldots$. Since the exponents resulting from Eq. (33) are non-commensurate, the secular terms are the terms that include any but only one of $e_{0}^{T}$ terms. The resulting six differential equations are of the following form:

$$
A^{\prime}\left(T_{1}\right)=\sigma_{i} A_{i}\left(T_{1}\right), \quad i=1, \ldots, 6
$$

where,

$$
\sigma_{i}=-\frac{(1-2 k) k \lambda+k\left(4+k-2 k \lambda+k^{2} \lambda\right) S_{i}^{2}+2\left(-2+2 k+k^{2}\right) S_{i}^{4}+k(8+5 k) S_{i}^{6}}{2 k(1+k)\left(\lambda-2 S_{i}^{4}-2 S_{i}^{6}\right)}
$$

The solution of Eq. (34) is

$$
A_{i}\left(T_{1}\right)=a_{i} e^{\sigma_{i} T_{1}}
$$

" $a_{i}$ "s are neither a function of $T_{0}$ nor $T_{1}$. Substituting Eqs. (32) and (36) in (29) and neglecting higher orders of $\epsilon$ results,

$$
B(\theta, \epsilon)=\sum_{i=1}^{6} a_{i} e^{\left(s_{i}+\sigma_{i} \epsilon\right) \theta}, \quad V(\theta, \epsilon)=\sum_{i=1}^{6} \frac{R}{k+1}\left(\frac{1}{s_{i}^{2}}-k\right) a_{i} e^{\left(s_{i}+\sigma_{i} \epsilon\right) \theta},
$$


Eq. (37) is the result of perturbation analysis. It gives an estimate of the effect of the rate of change of radius, $\epsilon$, on the solution of the system. Comparing Eq. (37) with Eq. (32) reveals that the rate of change of radius shifts the roots of the characteristic equation.

It remains to satisfy the boundary conditions to get the natural frequencies. This last step is similar to that of the previous section. The value of matrix $[M]$ is different from before due to the changes in the general solution. The exponent modifications, $\sigma_{i}$, appear in the coefficients matrix $[M]$. The values of $\lambda$ which make the matrix $[M]$ singular correspond to the natural frequencies of the system, and the dimensionless frequency parameter, $\lambda$, is only a function of $\epsilon$ and the arc length, $\alpha$.

The effect of normalized rate of change of radius, $\epsilon=R^{\prime} / R$, on the fundamental natural frequency of a clamped-clamped spiral beam is illustrated in Fig. 3. The length of the arc is $\alpha=\pi / 2$ and the three curves correspond to different stiffness

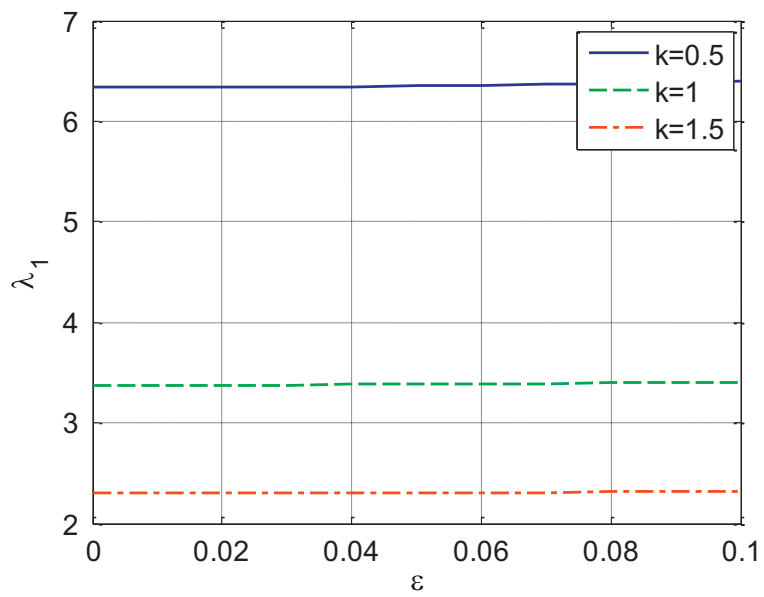

Fig. 3. Effect of derivative terms on fundamental natural frequency.

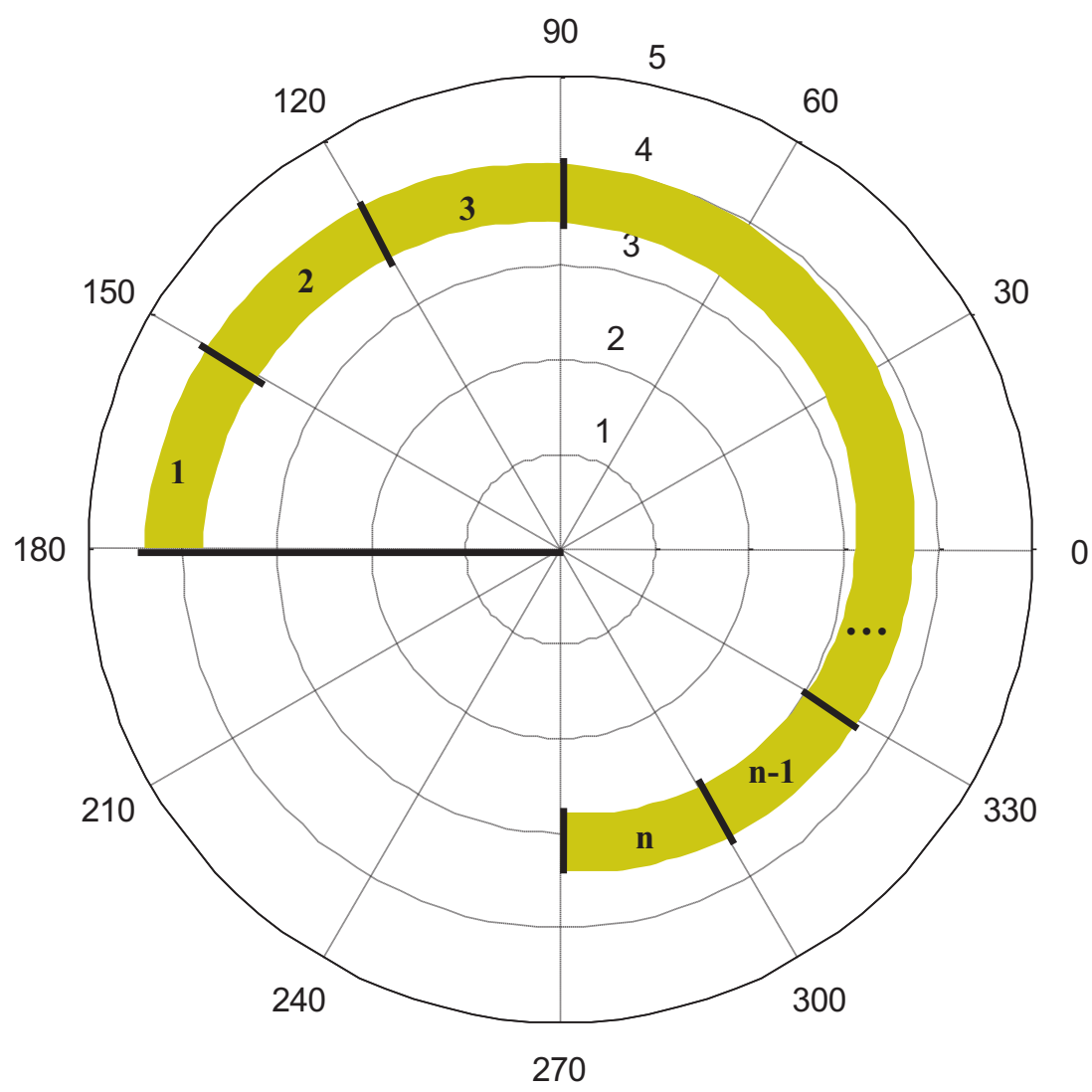

Fig. 4. Discretization of spiral. 
parameters. As can be seen in Fig. 3 the dimensionless natural frequency parameter is quite insensitive to the normalized rate of change of radius. We therefore conclude that it is reasonable to simply ignore their effect in calculating the natural frequencies of spiral beams Fig. 4.

\section{The effect of the slow variation of $R$ on the vibration of the spiral beam}

The above analysis allows us to exclude the effect of derivative terms in spiral beam vibrations. It now remains to evaluate the effect of the slow variation of $R$ on the natural frequencies of the spiral. Excluding the terms which contain $R^{\prime}$ in Eq. (27) yields

$$
\begin{aligned}
& V^{(4)}-k V^{\prime \prime}-(k+1) R B^{\prime \prime}=k \lambda V \\
& k R^{2} B^{\prime \prime}-R^{2} B+(k+1) R V^{\prime \prime}=0
\end{aligned}
$$

Considering that the rate of change of radius is small, we can divide the spiral into a number of segments and say that the radius is almost the same (constant) throughout each segment.

The general solution for each of the segments is already derived in Section 3. For the ith element we have

$$
B_{i}=\sum_{p=1}^{6} A_{i p} e^{S_{i p} \theta}, V_{i}=\sum_{p=1}^{6} \frac{R}{k+1}\left(\frac{1}{S_{i p}^{2}}-k\right) A_{i p} e^{S_{i p} \theta}
$$

$s_{i}$ is one of the six roots of characteristic equation for each of the pieces:

$$
s^{6}+2 s^{4}+\left(1-k R^{4} \mu\right) s^{2}+\mu R^{4}=0, \quad \mu=\frac{\rho A}{G J} \omega_{n}^{2}
$$

The above equation can be rewritten in terms of three dimensionless parameters, $k, \eta$ and $r$ :

$$
s^{6}+2 s^{4}+\left(1-k r^{4} \eta\right) s^{2}+r^{4} \eta=0
$$

where the dimensionless frequency parameter for a spiral beam, which is very similar to $\lambda$, is defined as

$$
\eta=\frac{\rho A}{G J} R_{0}^{4} \omega_{n}^{2}
$$

The radius dimensionless parameter is defined as

$$
r=\frac{R}{R_{0}}
$$

The boundary conditions of the spiral are the same as those of constant radius arc, meanwhile some matching conditions between elements are needed to relate the solution in each of the segments to the adjacent segment. Considering the problem from a degree of freedom aspect, each of the elements has 6 dofs, namely the $A_{i p} s$. If the spiral is composed of $n$ elements, there will be $6 n$ unknowns. There are six boundary conditions and six equilibrium and continuity equations can be written for each of the $n-1$ element interfaces. Therefore the number of equations will be the same as the number of unknowns. Since we have nondimensionalized our exponents the equilibrium and continuity constraints must be put into dimensionless form. The continuity and equilibrium nondimensionalized conditions at the interfaces are as follows.

$$
\begin{gathered}
\text { Deflection continuity } V_{i}\left(\theta_{\text {end }}\right) / R_{0}=V_{i+1} / R_{0}\left(\theta_{\text {start }}\right) \\
\text { Slope continuity } \quad V^{\prime}{ }_{i}\left(\theta_{\text {end }}\right) / R_{0}=V^{\prime}{ }_{i+1}\left(\theta_{\text {start }}\right) / R_{0} \\
\text { Twist angle continuity } \quad \beta_{i}\left(\theta_{\text {end }}\right)=\beta_{i+1}\left(\theta_{\text {start }}\right) \\
\text { Torsional moment equilibrium } \\
\frac{R_{0}}{G J} M_{z_{i}}\left(\theta_{\text {end }}\right)=\frac{R_{0}}{G J} M_{z_{i}+1}\left(\theta_{\text {start }}\right) \\
\text { Bending moment equilibrium } \quad \frac{R_{0}}{E I} M_{x_{i}}\left(\theta_{\text {end }}\right)=\frac{R_{0}}{E I} M_{x_{i}+1}\left(\theta_{\text {start }}\right) \\
\text { Shear force equilibrium } \quad \frac{R_{0}^{2}}{E I} Q_{i}\left(\theta_{\text {end }}\right)=\frac{R_{0}^{2}}{E I} Q_{i+1}\left(\theta_{\text {start }}\right)
\end{gathered}
$$

The expressions for the force and moments in terms of displacements can be distinguished from the boundary conditions expressed in Eqs. (8)-(10):

$$
M_{z}=\frac{G J}{R^{2}}\left(V^{\prime}+R \beta^{\prime}\right), \quad M_{x}=\frac{E I}{R^{2}}\left(R \beta-V^{\prime \prime}\right), \quad Q=E I\left(\frac{\beta^{\prime}}{R^{2}}(1+k)+\frac{-V^{(3)}+k V^{\prime}}{R^{3}}\right)
$$


The boundary conditions and the equilibrium and continuity constraints can be formed as a matrix relation:

$$
[M]_{6 n \times 6 n}\left[A_{11}, \ldots, A_{16}, A_{21}, \ldots, A_{26}, \ldots, A_{n 1}, \ldots, A_{n 6}\right]_{6 n \times 1}^{T}=0_{6 n \times 1}
$$

Eq. (43) yields trivial solution unless the determinant of $[M]$ is zero. The matrix $[M]$ is a function of $s_{i p}$ which in turn are functions of $\eta$. The eigenvalues, $\eta_{n}$, which make the determinant zero, are proportional to second power of natural frequencies of the system (refer to Eq. (41)).

The value of the first eigenvalue, $\eta_{1}$, of single turn spiral beams with varying radius for different beam radii and different number of elements are given in Fig. 5 . It can be seen that the natural frequencies become quite accurate after using only few sections. Since the formulation is nondimensionalized, the results in Fig. 5 are independent of $R_{0}$.

\section{Natural frequency calculation by Rayleigh's method}

An alternate approach to find the fundamental natural frequency of a spiral beam is Rayleigh's method. This approximate solution gives the natural frequency, for an assumed mode shape function. Choosing the static deflection shape of the spiral under its own weight as the mode shape is known to give an accurate estimate of the first natural frequency $[12,14]$. The main reason why we use this alternate approach to find the first natural frequency, is to compare it with the exact solutions. Although both methods are analytical and they share basic modeling assumptions, the agreement of their results confirms the accuracy of calculations.

We proceed applying Rayleigh's method in two steps. The first step is finding the static deformation shape. The deflection of the spiral beam under loads can be found by using Eqs. $(1,2)$ together with Eq. $(26)$ :

$$
\begin{gathered}
v^{\prime \prime}-\frac{R^{\prime}}{R} v-R \beta=-\frac{R^{2}}{E I_{x}} M_{x} \\
v^{\prime}+R \beta^{\prime}=\frac{R^{2}}{G J} M_{z}
\end{gathered}
$$

The parameters $M_{x}$ and $M_{z}$ are the bending moment and the torsional moment caused by the gravitational load. The moments are zero at the free end of the beam. At a cross section, $\theta$ radians from the clamed end (refer to Fig. 6), the

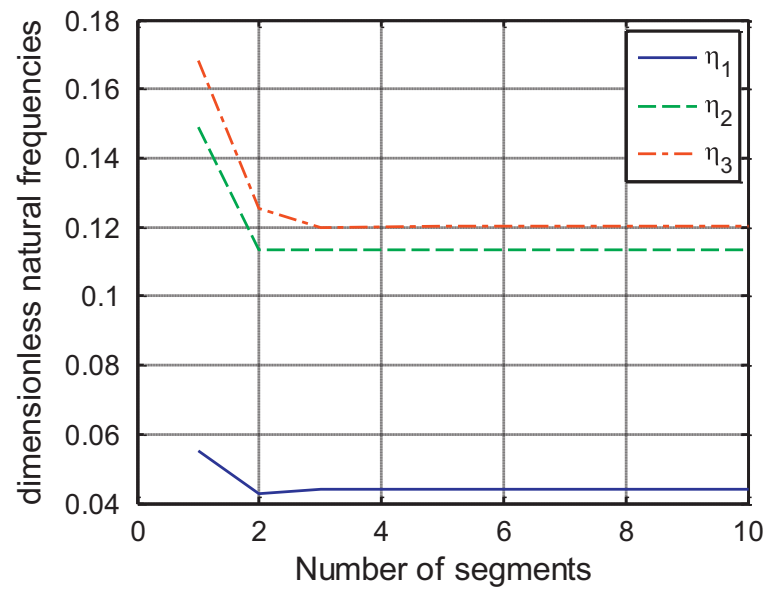

Fig. 5. Effect of radius variations and number of elements on eigenvalues, $\boldsymbol{k}=1, \epsilon=-0.1$.

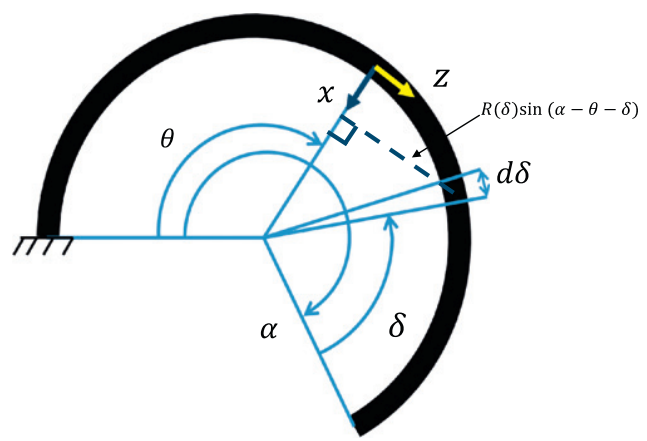

Fig. 6. Parameters used to find gravitational moments. 
gravitational bending moment and torsional moment are:

$$
\begin{gathered}
M_{x}(\theta)=-m g \int_{0}^{\alpha-\theta} R^{2}(\delta) \sin (\alpha-\theta-\delta) d \delta \\
M_{z}(\theta)=m g \int_{0}^{\alpha-\theta} R(\delta)[R(\theta)-R(\delta) \cos (\alpha-\theta-\delta)] d \delta
\end{gathered}
$$

The values of $M_{x}$ and $M_{z}$ from Eqs. ((46), (47)) can be used in Eqs. ((44), (45)). The latter equations are next numerically integrated to result in the static deflection shapes. We call the bending and torsion static deformations $\psi_{v}$ and $\psi_{\beta}$, respectively.

The second step in Rayleigh's method is finding the natural frequency for the assumed mode shapes. We assume $\psi_{v}$ and $\psi_{\beta}$ as the mode shapes and we write the kinetic and elastic energies as

$$
\begin{gathered}
T=\frac{1}{2} \Gamma^{2}(t) m \int_{0}^{\alpha} \psi_{\nu}^{2} R(\theta) \sqrt{1+\epsilon^{2}} d \theta \equiv \frac{1}{2} \Gamma^{2}(t) \Phi_{T} \\
U=\frac{1}{2} \Gamma^{2}(t) G J \int_{0}^{\alpha}\left(\frac{1}{k R^{4}}\left(R \psi_{\beta}-\psi^{\prime \prime}{ }_{v}+\epsilon \psi^{\prime}{ }^{\prime}\right)^{2}+\frac{1}{R^{4}}\left(R \psi^{\prime}{ }_{B}+\psi^{\prime}{ }^{\prime}\right)^{2}\right) R \sqrt{1+\epsilon^{2}} d \theta \equiv \frac{1}{2} \Gamma^{2}(t) \Phi_{U}
\end{gathered}
$$

The temporal part of the solution is noted by $\Gamma(t)$. The corresponding natural frequency of the spiral is then

$$
\omega_{n}^{2}=\frac{\Phi_{U}}{\Phi_{T}}
$$

We can also factor out $m$ from $\Phi_{T}\left(\Phi_{T}=m \bar{\Phi}_{T}\right)$ and $G J$ from $\Phi_{U}\left(\Phi_{U}=G J \Phi_{U}\right)$ to evaluate the dimensionless frequency parameter:

$$
\eta=\frac{m}{G J} R_{0}^{4} \omega_{n}^{2}=R_{0}^{4} \frac{\bar{\Phi}_{U}}{\bar{\Phi}_{T}}
$$

Since the static deflection shape has been chosen as the shape function, $\eta$ is an approximate of the first dimensionless natural frequency. According to [15] the approximate natural frequency should be larger than the exact value of the fundamental frequency. We will check this condition while discussing the Rayleigh's results in Section 6.2.

\section{Results}

In the possible application of spirals to energy harvesting a key feature is that the natural frequency of the substrate should be as close as possible to the ambient excitation frequency, which is typically less than $100 \mathrm{~Hz}$. We therefore, start with the natural frequency relations. As mentioned earlier, the dimensionless natural frequency parameter , $\eta_{i}$, is a function of three dimensionless parameters: $\alpha, k$ and $\varepsilon$. The influence of each of the parameters on $\eta$ is explained in the following. It must be noted that all the sub-sections except Section 7.2 discuss results of the exact solution. The Rayleigh's method is used only for verification. In all of the following case studies the structure is clamped at the large radius end and is free at the small radius end. Clamping the spiral at its large radius end is advantageous compared to clamping at the small radius side since it results in a smaller stress concentration factor at the joint.

\subsection{The relation between the natural frequencies and the arc length}

Fig. 7 illustrates the first six roots of the characteristic equation as functions of the spiral length, $\alpha$. Two of the six lines appear to be flat which suggests there are two natural frequencies which do not change as the arc length increases. This is of course unrealistic. The higher length corresponds to more mass and less stiffness both for bending or torsion mechanisms which should translate to less natural frequency. The problem is rooted in the fact that we did not directly use the governing relation equation (18), but we differentiated it (Eq. (19)) twice. The double differentiation has introduced two non-physical answers. Our solution method ensures that left hand side (LHS) of Eq. (19) equals zero. Although every solution of Eqs. (18) satisfies (19), the reverse is not true. For example, if the LHS of Eq. (18) equals any linear function of $\theta$, Eq. (19) is satisfied but Eq. (18) is violated. The solutions which satisfy Eq. (19) but not Eq. (18) are the non-physical solutions. It is therefore concluded that the four "decreasing" curves in Fig. 7 correspond to the first four natural frequencies. The Rayleigh's result in the next sub-section verifies this distinction of the natural frequencies from non-physical eigen-solutions.

Once the dimensionless natural frequency parameter, $\eta_{i}$, is read from Fig. 7 , the corresponding natural frequency can be evaluated from:

$$
\omega_{i}=R_{0}^{2} \sqrt{\frac{G J}{\rho A} \eta_{i}}
$$




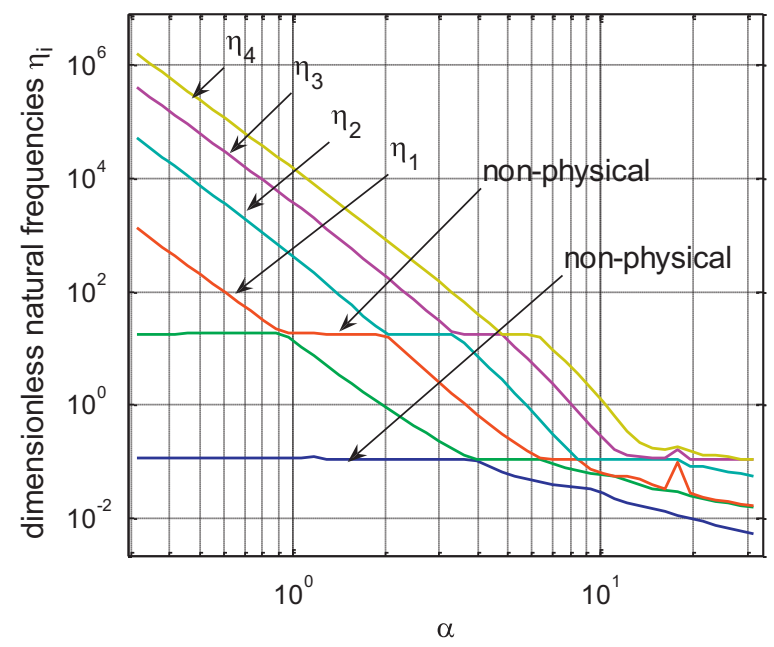

Fig. 7. The first four natural frequency parameters; $\boldsymbol{k}=1, \boldsymbol{\epsilon}=-0.1 / 2 \pi$.

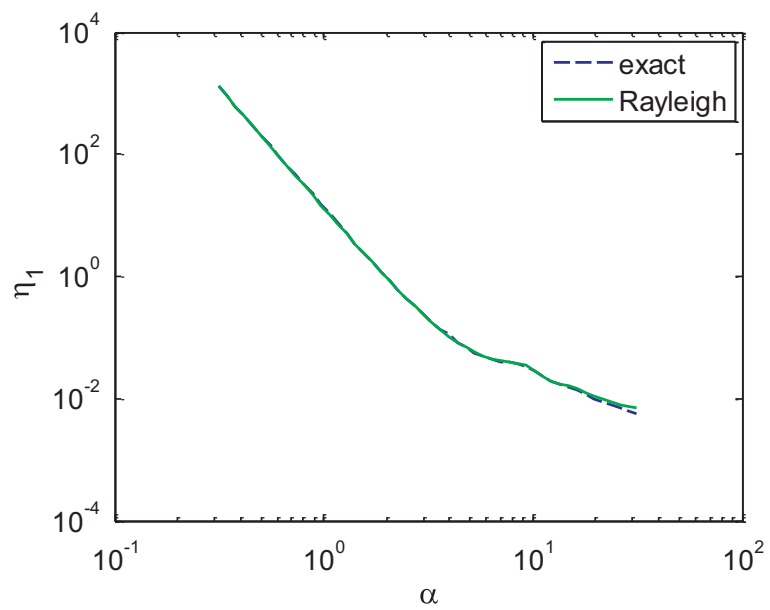

Fig. 8. Rayleigh vs. exact fundamental frequency; $\boldsymbol{k}=1, \boldsymbol{\epsilon}=-0.1 / 2 \pi$.

The drop in the natural frequencies makes the spiral geometry a good candidate for reducing the natural frequencies of MEMS harvesters. The fundamental natural frequency of a five turn spiral is about $1 / 7$ of the fundamental natural frequency of a single turn. Since in the longer spirals, the radius of the final turns are noticeably smaller than the first ones, the rate of frequency drop flattens as the number of turns increases.

\subsection{Results of Rayleigh's method}

As illustrated in Fig. 8, the results from Rayleigh's method are in close agreement with the exact fundamental natural frequency. The difference becomes visible only for large number of turns (more than 4 turns). The Rayleigh frequency is always larger than the exact, which agrees with the condition in [15]. This matching between the results of two different methods confirms the accuracy of calculations. The accuracy of underlying modeling assumptions is not confirmed by this agreement and can be tested by experimental modal analysis.

Since in Rayleigh's method we are guessing the mode shapes, the calculations involved in this method are significantly less cumbersome compared to the calculations in the exact solution. The fundamental frequency is well approximated by Rayleigh's method, so this method is a practical candidate for finding the first resonance frequency.

\subsection{The relation between the natural frequencies and $k$}

The graphs in Fig. 7 are only valid for the case $k=1$ and $\epsilon=-0.1 / 2 \pi$. Fig. 9 illustrates the effect of the variation of $k$ on the first two natural frequencies. The natural frequencies of the short arcs are quite sensitive to the torsion-bending 
(a)

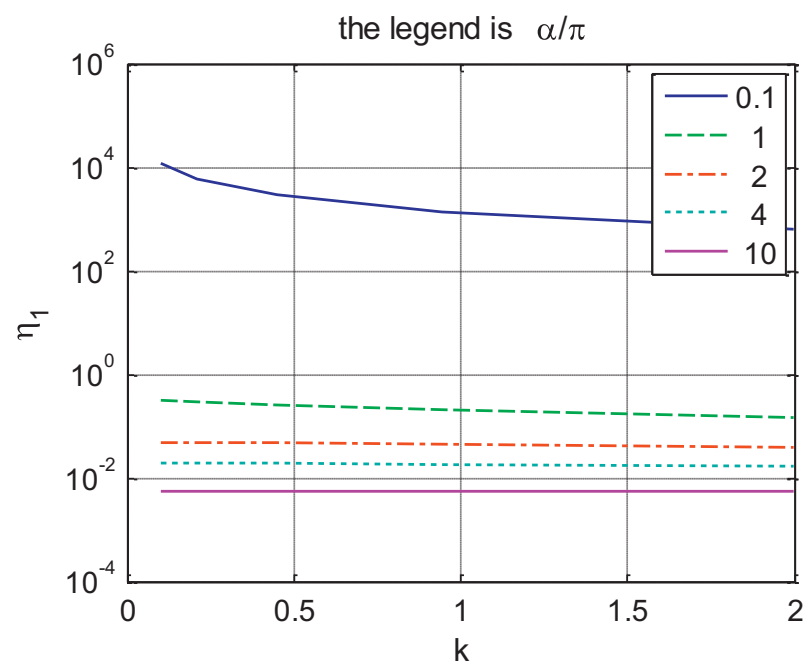

(b)

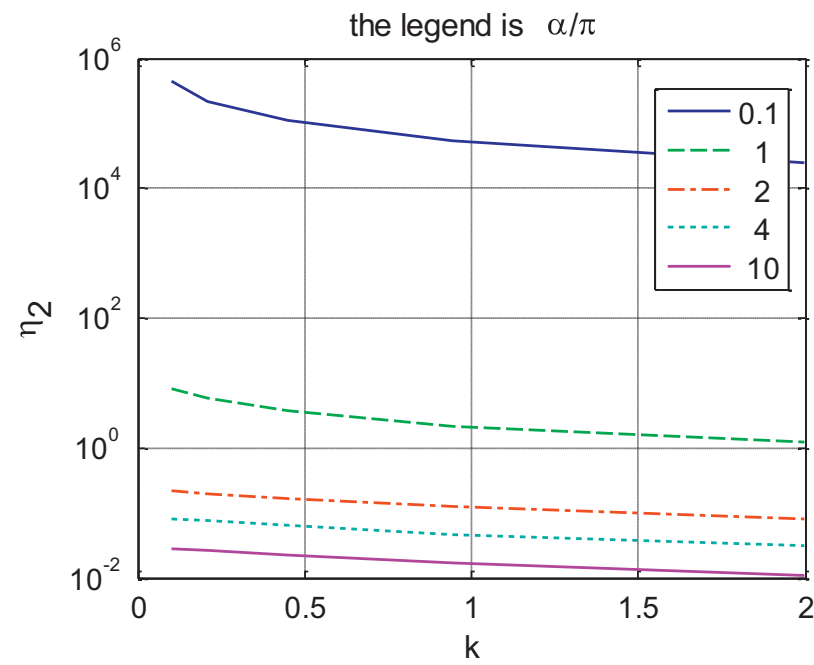

Fig. 9. The effect of $\boldsymbol{k}$ on natural frequencies; the legend is $\boldsymbol{\alpha} / \boldsymbol{\pi}$. (a) first mode, (b) second mode.

(a)

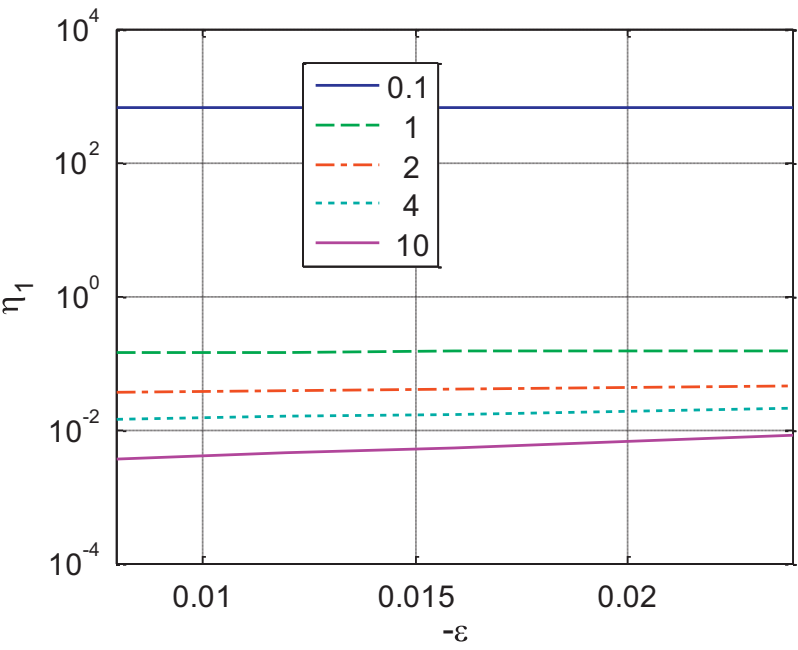

(b)

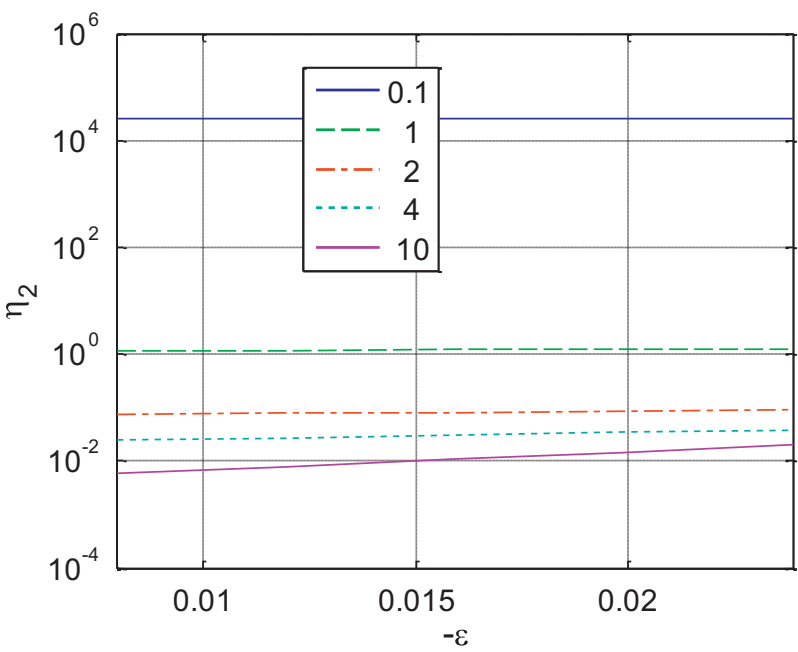

Fig. 10. The effect of $\boldsymbol{\varepsilon}$ on natural frequencies; $\boldsymbol{k}=2$, the legend is $\boldsymbol{\alpha} / \boldsymbol{\pi}$ (a) first mode, (b) second mode.

stiffness ratio, but as the arc length increases the sensitivity decreases. Note that the independence of $\eta$ from $k$ does not at all imply the independence of frequency from the material properties. The torsional stiffness always appears at the last step of evaluating the natural frequency (Eq. (51)). If variation of $k$ does not affect the frequency, it means that the frequency is unrelated to bending stiffness. The fact will be explained in sec 6.5. The vibrations of longer arcs are mostly torsional and therefore mostly the torsional stiffness, GJ, affects the frequencies.

\subsection{The relationship between the natural frequencies and $\varepsilon$}

It is more desirable to have the structure spiral in than out. A spiraling out structure has its smallest radius at the clamp point. This translates to having the highest stress concentration factor at the clamp, which results in lower strength. The natural frequencies of a spiral beam have been plotted for different values of $\varepsilon$ in Fig. 10. $\varepsilon$ is considered negative, i.e. the structures spiral inwards.

Consider two spirals with the same number of turns but with different rates of change of the radii. The final turns of the spiral with larger $\varepsilon$ have a smaller radii than its first turns. So that spiral will be stiffer and it is expected to have higher natural frequencies compared to the spiral with smaller $\varepsilon$. It can be seen from Fig. 10 that the fundamental natural frequency of long spirals have direct relationships with the absolute value of $\varepsilon$, which agrees with the expectations. The 
fundamental natural frequency of short spirals is, in contrast, independent from $\varepsilon$. The radii of short structures are almost constant all along the spirals and therefore there is not a noticeable difference between the natural frequencies of short structures with different rates of changes of radii.

Figs. 9 and 10 not only show the trend of variation of natural frequencies with changes in $k$ and $\varepsilon$ but also can be used as tools to help calculate natural frequencies for a structure with arbitrary values of $k$ and $\varepsilon$.

\subsection{Mode shapes}

The resulting mode shapes for $k=1, \epsilon=-0.3 / 2 \pi, \alpha=2 \pi$ and $n=10$ are depicted in Fig. 11 for a clamped-free spiral. The mode shapes of a five turn clamped-free spiral with $k=2, \epsilon=-0.1 / 2 \pi, \alpha=10 \pi$ and $n=10$ are depicted in Fig. 12 . The mode shapes confirmed that the essential boundary conditions and the continuity conditions are satisfied.

There are three plots corresponding to each mode shape in Figs. 11 and 12. The 2D (two-dimensional) plots illustrate the bending and torsional mode shapes as a function of $\theta$. The out of plane deformation has been re-illustrated in a 3D plot to help visualize the mode shapes. The 3D graphs therefore, contain the same data presented in the first $2 \mathrm{D}$ graphs.

\subsection{Torsional vs. bending vibrations}

The piezoelectric energy harvesters are commonly made as Unimorph or Bimorph structures. The structures are at least two layered; the bottom layer is "substructure" which supports the piezoelectric layer on top (Fig. 13). The spiral structure

(a.l)

$$
\eta_{n}=0.044606
$$

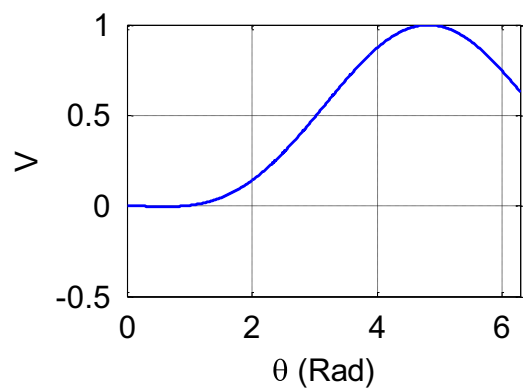

(b.l)

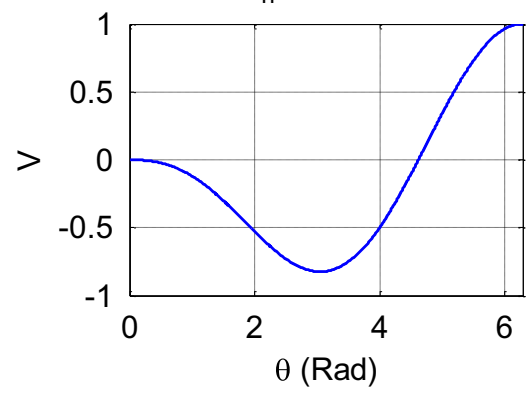

(c.l)

$$
\eta_{n}=0.58919
$$

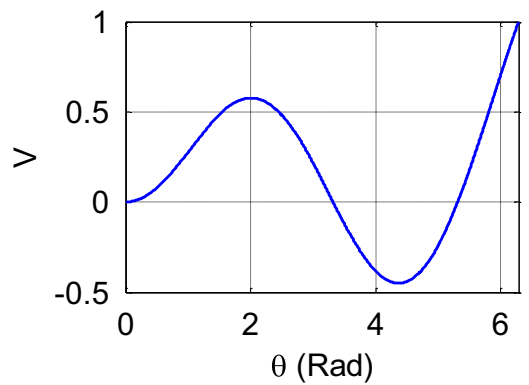

(a.II)

$\eta_{n}=0.044606$

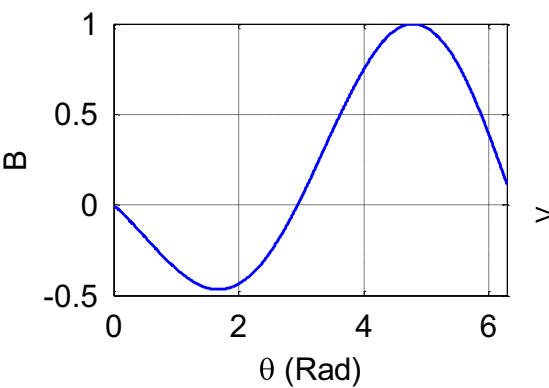

(b.II)

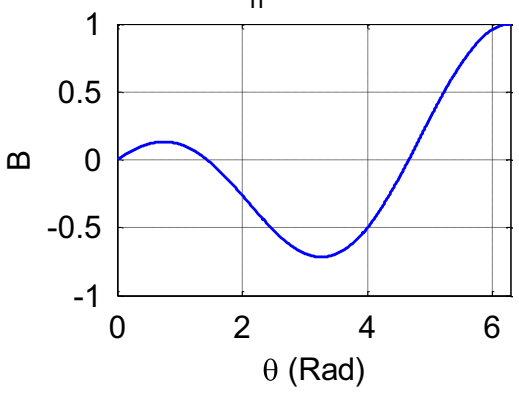

(c.II)

$\eta_{n}=0.58919$

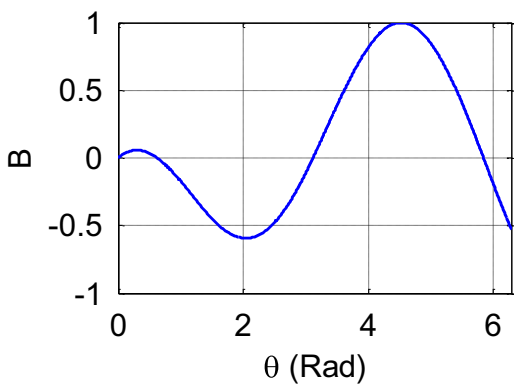

(a.III)

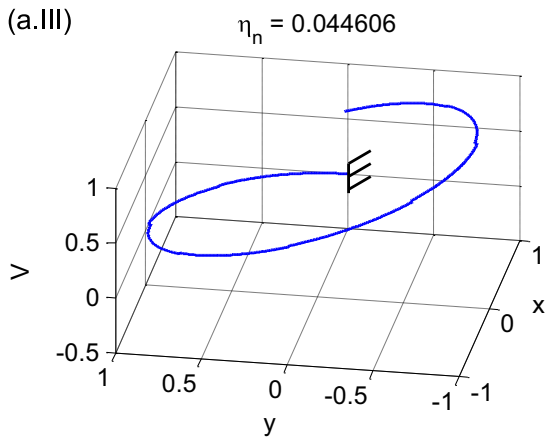

(b.III)

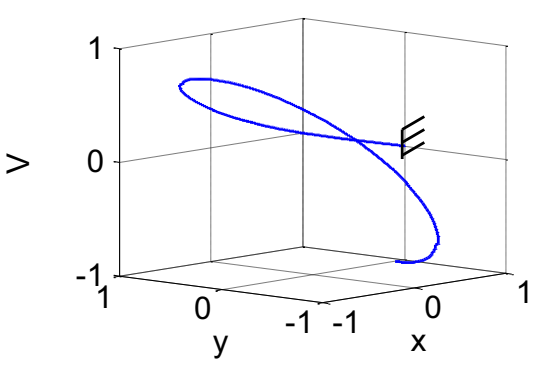

(c.III)

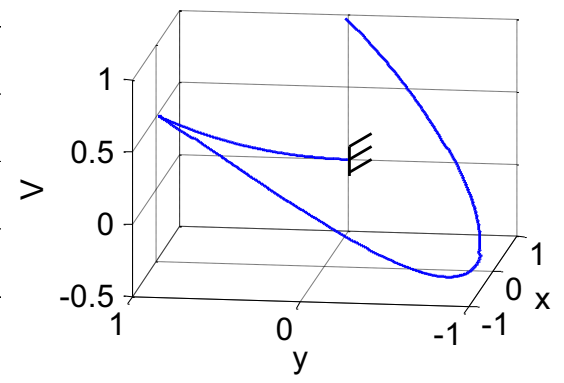

Fig. 11. Mode shapes of a full turn spiral: (a) first mode shape, (b) second mode shape, (c) fourth mode shape. (I) Bending function, (II) torsion function, (III) modal deformation shape. 
(a.I)

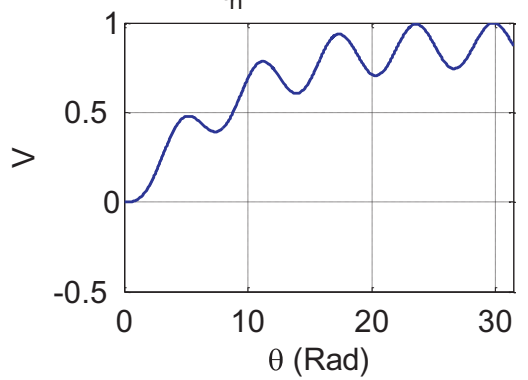

(b.l)

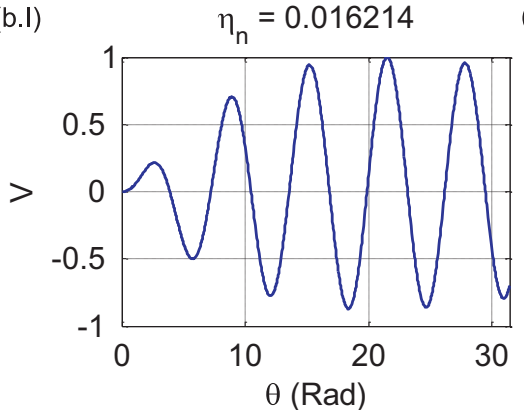

(c.l)

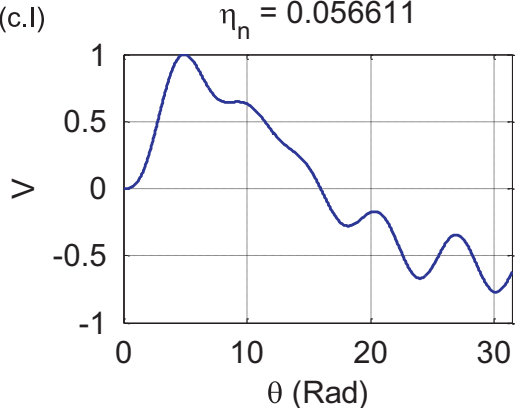

(a.II)

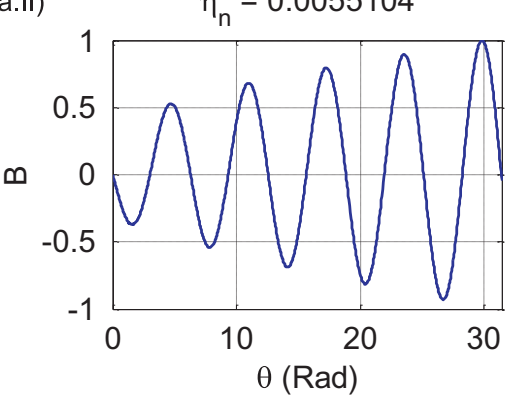

(b.II)

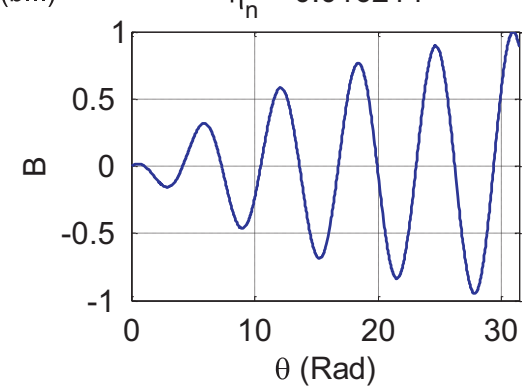

(c.II)

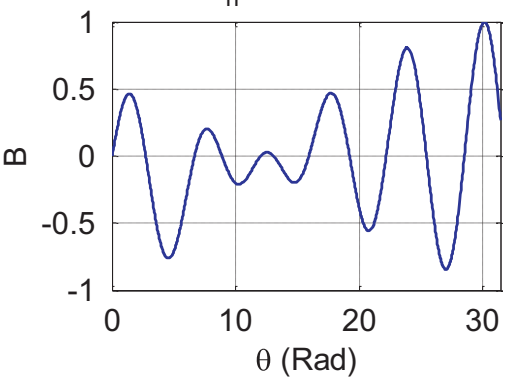

(a.III) $\eta_{n}=0.0055104$

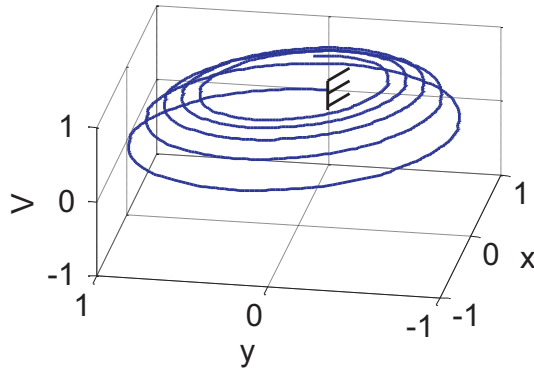

(b.III)

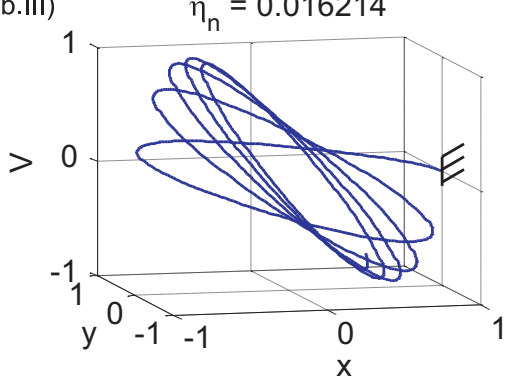

(c.III)

$$
\eta_{n}=0.056611
$$

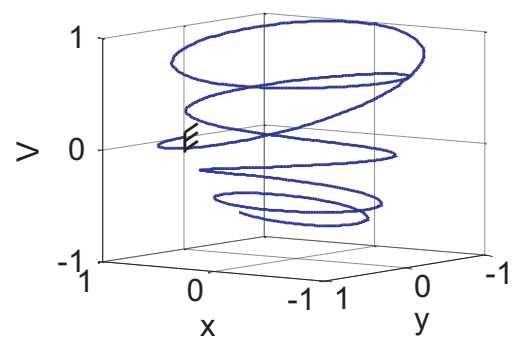

Fig. 12. mode shapes of a five turn spiral. (a) first Mode shape, (b) second mode shape, (c) fourth mode shape. (I) bending function, (II) torsion function, (III) modal deformation shape.

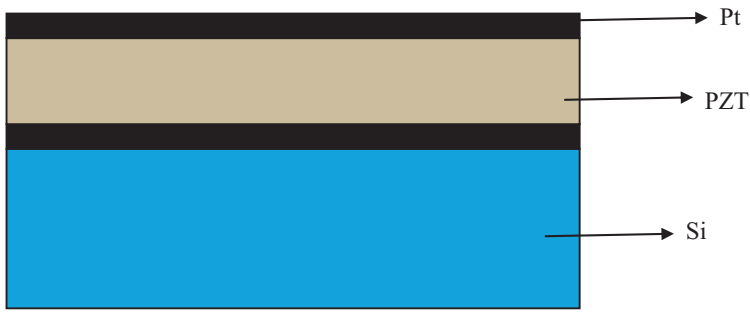

Fig. 13. Unimorph cross section.

can be used as the substructure and it can be made out of silicon. Three layers, two thin platinum layers sandwiching the piezoelectric layer are deposited on the substrate. The platinum layers are the electrodes and are used for polarizing the Piezo-material in the first place. In addition, the normal stresses in the Piezo-layer generate electric field in the thickness direction and therefore the same top and bottom electrodes are used to harvest the electrical energy resulted from the bending. The torsional stresses also result in electrical energy. However for common piezoelectric materials, the polarization axis, the torsional stress vector and the generated electric field vector are mutually orthogonal. Harvesting energy from torsional stress therefore requires more complicated electrode configuration (the four opposing faces of the cross section should form two pairs of electrodes). Thus in practical energy harvesting application bending vibrations can be easily harvested but torsional vibrations cannot .To be able to investigate the abundance of bending deformation of the structure we evaluate the bending/torsional energy ratio as the bending deflection energy of the structure divided by the 
(a)

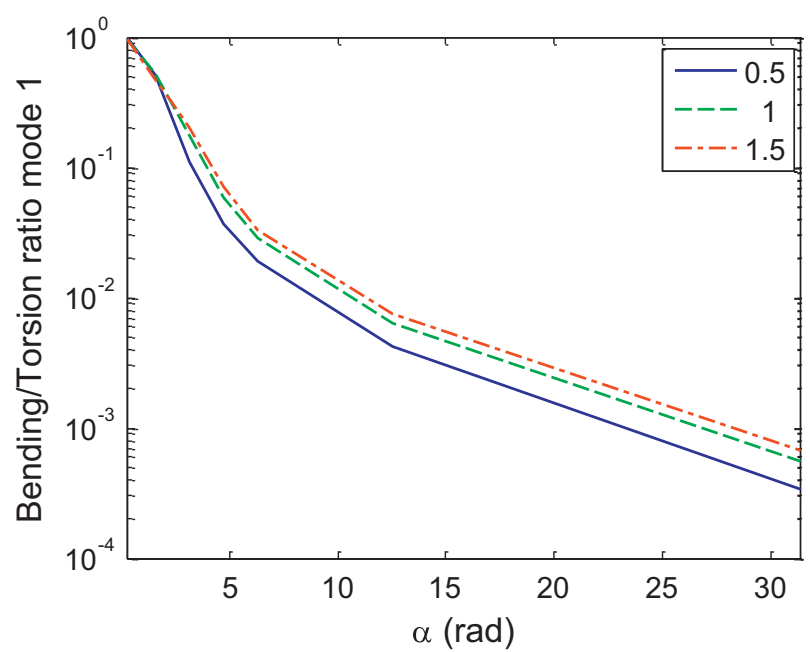

(b)

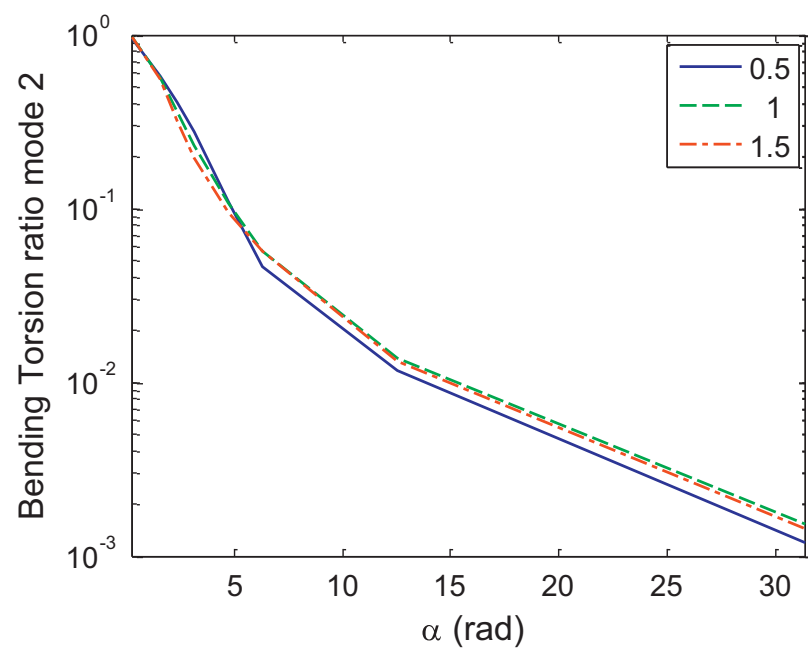

Fig. 14. Bending/torsional energy index: (a) first mode, (b) second mode; $\boldsymbol{\epsilon}=-0.1 / 2 \pi$, the legend is the value of $\boldsymbol{k}$.

torsion deflection energy. This index is calculated for each mode shape and indicates whether the vibration is dominantly torsional or bending.

The bending/torsional energy index plotted in Fig. 14 indicates that although the vibration of the very short spiral beams are dominantly bending, as the spiral gets longer the vibration becomes dominantly torsional. We are mostly interested in longer spirals due to their low natural frequency. Unfortunately there is not that much bending in the structure for a structure longer than half a turn and therefore a complicated electrode configuration is needed for energy harvesting from the spiral beams.

\section{Conclusion}

Here we proposed an analytical method to calculate the natural frequencies and the mode shapes of spiral beams. We showed that the effect of derivative terms on the structural dynamics can be neglected. Thus the structure was approximated by many constant radius sections joined together, to tackle the problem of slowly changing coefficients. The piecewise continuous solution converged with relatively few elements. The dimensionless natural frequencies depend on the stiffness ratio, the rate of change of radius and the total angle. The effect of each parameter on natural frequency was evaluated through a non-dimensional analysis. The natural frequency results were verified using Rayleigh's method. The resulting mode shapes of two different spirals were depicted for illustration. It was shown that the vibration of practical spiral beams is dominantly torsional and therefore more complicated electrode configuration is necessary for energy harvesting. This study paves the way for a more comprehensive study of the coupled electromechanical vibrations which gives the output power from the device.

\section{Acknowledgements}

This work was performed under the support of the US Department of Commerce, National Institute of Standards and Technology, Technology Innovation Program, Cooperative Agreement Number 70NANB9H9007.

The first author would like to thank Mr. Arash Bahrami for the useful conversations on the subject.

\section{References}

[1] S. Anton, H. Sodano, A review of power harvesting using piezoelectric materials (2003-2006), Smart Materials and Structures 16 (2007) 1.

[2] W.J. Choi, Y. Jeon, J.H. Jeong, R. Sood, S.G. Kim, Energy harvesting MEMS device based on thin film piezoelectric cantilevers, Journal of Electroceramics 17 (2006) 543-548.

[3] H. Hu, H. Xue, Y. Hu, A spiral-shaped harvester with an improved harvesting element and an adaptive storage circuit, IEEE Transactions on Ultrasonics, Ferroelectrics and Frequency Control 54 (2007) 1177-1187.

[4] A.E.H. Love, A Treatise on the Mathematical Theory of Elasticity, 4th ed., Dover, New York, 1944.

[5] T.C. Chang, E. Volterra, Upper and lower bounds for frequencies of elastic arcs, The Journal of the Acoustical Society of America 46 (1969) 1165-1174.

[6] T.M. Wang, Fundamental frequency of clamped elliptic arcs for vibrations the plane of initial curvature, Journal of Sound and Vibration 42 (1975) 515-519.

[7] T. Irie, G. Yamada, I. Takashashi, The steady state out-of-plane response of a Timoshenko curved beam with internal damping., Journal of Sound and Vibration 71 (1980) 145-156. 
[8] C.S. Huang, Y.P. Tseng, S.H. Chang, Out-of-plane dynamic responses of non-circular curved beams by numerical Laplace transform, Journal of Sound and Vibration 215 (1998) 407-424.

[9] C.S. Huang, Y.P. Tseng, S.H. Chang, C.L. Hung, Out-of-plane dynamic analysis of beams with arbitrarily varying curvature and cross-section by dynamic stiffness matrix method, International Journal of Solids and Structures 37 (2000) 495-513.

[10] E. Tufekci, O.Y. Dogruer, Exact solution of out-of-plane problems of an arch with varying curvature and cross section, ASCE Journal of Engineering Mechanics 132 (2006) 600-609.

[11] I.U. Ojalvo, Coupled twist-bending vibrations of incomplete elastic rings, International Journal of Mechanical Sciences 4 (1962) 53-72.

[12] D.J. Inman, Engineering Vibration, 3rd ed., Prentice Hall, New Jersey, 2007.

[13] A.H. Nayfeh, Introduction to perturbation techniques, Wiley, New York, 1981.

[14] S.S. Rao, Vibration of Continuous Systems, John Wiley and Sons, 2007.

[15] L. Meirovitch, Analytical Methods in Vibration, Mecmillan Company, London, 1967. 